\title{
Analysis of Surface Electromyography Signals in Transtibial Amputees in Fatigue and Non Fatigue Conditions
}

\author{
Authors \\ Dr Priyadarshini.C.S ${ }^{1}$, Dr Debbie Aishwarya Sathya ${ }^{2}$ \\ ${ }^{1}$ Madras Medical College, Government Institute of Rehabilitation Medicine, Chennai, India \\ Email: aishdeepthi@yahoo.com \\ ${ }^{2}$ Sree Balaji Medical College, Chennai, India
}

\begin{abstract}
Aim: To analyse rectus femoris muscle activity of subjects with transtibial amputation in sound and amputated leg during isometric contraction until fatigue using surface electromyography signals (sEMG) and multifractal features.

Method and Materials: Eight subjects with unilateral transtibial amputation participated in this study and surface Electromyogram (sEMG) signals were recorded from rectus femoris muscle using standard experimental protocol. The signals were segmented into epochs and each epoch was subjected to multifractal detrended moving average algorithm (MFDMA) algorithm. Four multifractal features namely, degree of multifractality, maximum and minimum Hurst exponent, strength of multifractality were extracted and compared with median frequency and root mean square trends in (a) sound and amputated leg $(b)$ nonfatigue and fatigue condition. Statistical analysis used: 2-way ANOVA was used to analyse the multifractal features in nonfatigue and fatigue condition for both sound and amputated leg.

Results: The results show that sEMG signals exhibit multifractal behavior in both the sound and amputated leg. The multifractal features are clearly distinguishable in nonfatigue and fatigue condition for both sound and amputated leg ( $p$-value $<0.001)$ and proved to be better than the conventional features such as median frequency and root mean square.

Conclusion: The variations due to small and large fluctuations were distinctly seen in multifractal features as compared to conventional features. Hence this technique of multifractal analysis will prove useful as a biomarker to reduce or prevent muscle weakness and fatigue.
\end{abstract}

Keywords: Transtibial amputation, muscle fatigue, surface electromyography.

\section{Introduction}

There is a steady increase of amputation of the lower limb due to accidents and diseases. Transtibial or below knee amputation involves removal of leg muscles but the quadriceps femoris muscle which is on the front of the thigh is intact ${ }^{[1]}$. Quadriceps femoris muscle consists of 4 parts rectus femoris (RF), vastus medialis, vastus intermedius and vastus lateralis of which rectus femoris is superficial $^{[2]}$. Prolonged and sustained fatigue in the lower limb muscles can cause damage and adversely impact movement in the sound and residual lower limbs of amputees.

Analysis of muscle fatigue by surface electromyography $(\mathrm{sEMG})^{[3]}$ is performed using conventional signals such as time- root mean square (RMS) and frequency- (median frequency). 
However, these conventional signal processing techniques assume that the signals are stationary or linear within the small time interval, whereas the sEMG signals are nonlinear, nonstationary and exhibit self-similar fractal behaviour ${ }^{[3],[4]}$. Hence nonlinear and nonstationary techniques such as entropy, complexities and fractal dimensions appear to be suitable for sEMG analysis ${ }^{[5]}$. The analysis of muscle fatigue in persons with transtibial amputation using advanced sEMG analysis is not fully established in the literature, hence the need to conduct the present study.

The objective of this study was to analyse the muscle activity of transtibial amputees recorded from the rectus femoris muscle in sound and amputated leg during isometric contraction until fatigue. The signals were analysed using multifractal detrended moving average algorithm MFDMA and multifractal features are extracted to analyse and establish (a) muscle activity of sound and amputated leg (b) muscle activity in nonfatigue and fatigue condition.

\section{Material and Method}

\section{2a. Subjects}

Eight unilateral subjects with transtibial amputation participated in this study conducted at Government Institute of Rehabilitation Medicine, Chennai. Inclusion criteria were persons with unilateral transtibial amputation, age between 20- 60 years, willingness to cooperate in the study, no problems in the sound leg such as fracture and deformities. Exclusion criteria were persons with recent myocardial infarction, systemic hypertension, heart disease, congenital amputation and unmotivated persons.

\section{2b. Signal Acquisition}

The experiment was conducted in accordance with Declaration of Helsinki and approved by the Institute Ethics Committee. A written informed consent was taken for all subjects. The EMG signals were recorded using Biopac MP36 bio amplifier system (CMRR $110 \mathrm{~dB}$; sampling rate $10 \mathrm{kHz}$; Gain 1000). The electrodes were placed on the middle of the rectus femoris muscle, and the reference electrode was placed medial to the tibial tuberosity ${ }^{[6]}$ as shown in fig 1 . The subjects were advised to lay flat on a firm platform and perform maximum isometric contraction until fatigue. The signals were recorded for the entire duration of the experiment until the subject was no longer able to sustain the contraction or experience extreme pain. The signals were segmented into epochs and each epoch was subjected to multifractal detrended moving average algorithm MFDMA algorithm. Four conventional multifractal features namely, degree of multifractality, maximum and minimum Hurst exponent, strength of multifractality (SOM) were extracted and compared with median frequency (MDF) and root mean square (RMS) trends in (a) sound and amputated leg (b) nonfatigue and fatigue condition.

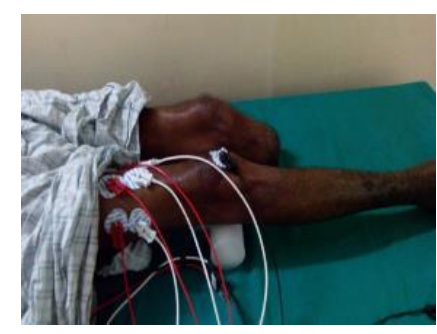

Fig 1: Electrode placement for quadriceps muscle including rectus femoris part

\section{2c. Protocol}

\section{Multifractal DMA algorithm}

The multifractal fluctuation detrended moving average (MFDMA) was used for analyzing nonstationary time series by computing the average and subtracting the original series with the moving average function ${ }^{[7]}$. The time series are represented in the form of fluctuation function $\left(F_{q}\right)$ for different orders $\mathrm{q}$ ranging from -10 to +10 . The fluctuation function are representation of the power-law scaling for large segments (unfolds them with different order) and are represented as a function of segment (s) of the time series and generalized Hurst exponent $\left(H_{q}\right)$. Four features extracted using the generalized Hurst exponent function and multifractal spectrum based on from earlier work ${ }^{[8]}$. 
- $\mathrm{H}_{\mathrm{MAX}}$ - Maximum value of Hurst exponent for negative order where q equals -10

- $\mathrm{H}_{\mathrm{MIN}}$ - Minimum value of Hurst exponent for positive order where q equals +10

- DOM - Degree of multifractality is the difference between $\mathrm{H}_{\mathrm{MAX}}$ and $\mathrm{H}_{\mathrm{MIN}}$

- SOM - Strength of multifractality is the difference between extremes in the multifractal spectrum

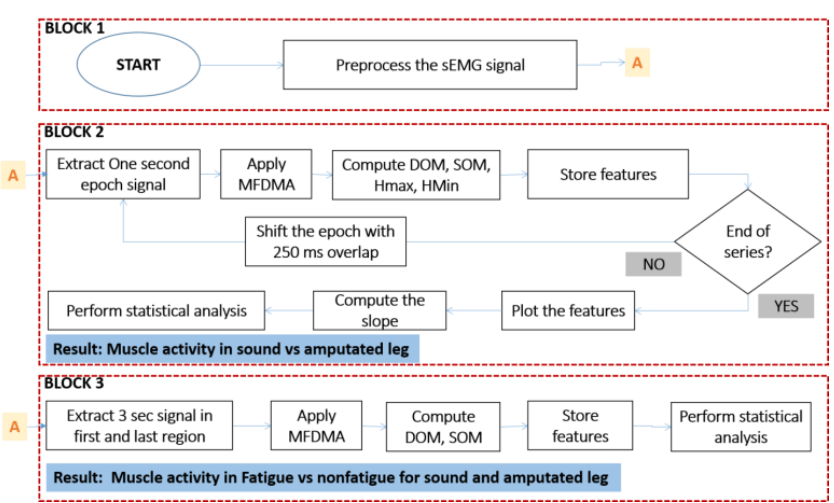

Fig 2: shows the workflow for processing the sEMG signal using multifractal technique. Block 2 shows the progressive analysis of isometric contraction by extracting epochs (1 second with overlap of 250ms) and computing MFDMA at each epoch. Block 3 show the workflow for the analysis of nonfatigue and fatigue conditions using three second segment from the first (nonfatigue) and last (nonfatigue) regions of the sEMG signal and subjected to MFDMA algorithm and compared with RMS and MDF.

\section{Results}

sEMG signals were analysed for rectus femoris component of quadriceps femoris muscle in eight amputated and eight sound legs. The demographic details of the subjects is shown in Table 1.

Table 1 Demographics data of Transtibial amputees

\begin{tabular}{|l|c|c|c|c|c|}
\hline Subject & Sex & $\begin{array}{c}\text { Amputated } \\
\text { leg }\end{array}$ & $\begin{array}{c}\text { Cause of } \\
\text { Amputation }\end{array}$ & $\begin{array}{c}\text { Age } \\
\text { (yrs) }\end{array}$ & $\begin{array}{c}\text { Amputated } \\
\text { Duration } \\
\text { (yrs })\end{array}$ \\
\hline S1 & Male & Right & Accident & 24 & 4 \\
\hline S2 & Male & Right & Accident & 26 & 1.5 \\
\hline S3 & Male & Right & Diabetic & 55 & 2 \\
\hline S4 & Female & Left & Vascular & 53 & 2 \\
\hline S5 & Male & Right & Accident & 28 & 0.5 \\
\hline S6 & Male & Left & Diabetic & 60 & 1.5 \\
\hline S7 & Male & Left & Diabetic & 60 & 0.5 \\
\hline S8 & Male & Left & Accident & 37 & 6 \\
\hline
\end{tabular}

\section{3a. sEMG signals}

The sEMG signals recorded from rectus femoris during isometric contraction in the sound leg and amputated leg are shown in Fig. 3. The amplitude of the sEMG signals recorded from the sound leg clearly showed higher muscle activity as compared to the amputated leg. The amplitude varied between $\pm 0.1 \mathrm{mv}$ and $\pm 0.05 \mathrm{mv}$ in the sound and amputated leg. The duration of contraction until fatigue (endurance time) in sound leg of representative subject is about 30 seconds and that of amputated leg is about 20 seconds. The average endurance time in the sound leg is observed to be lower than the amputated leg in majority of cases. This may be due to fatigability in amputated leg. The average contraction time recorded from the sound and amputated legs are $25.5 \mathrm{sec}$ and $34.5 \mathrm{sec}$ respectively. Based on Fig 3, it is observed that the sEMG signals at end of endurance time (fatigue condition) are not clearly distinguishable from start of experiment (nonfatigue region).

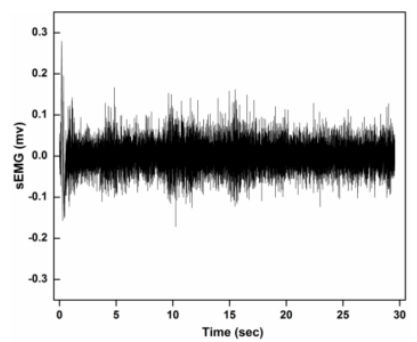

(a)

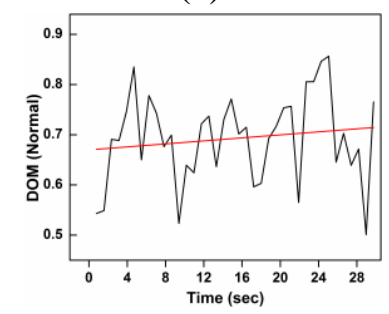

(c)

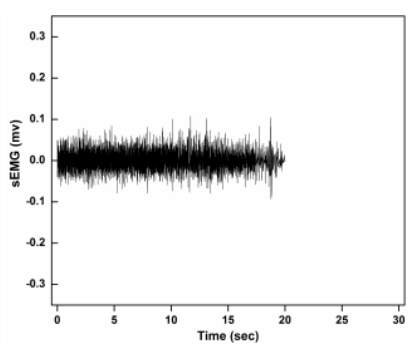

(b)

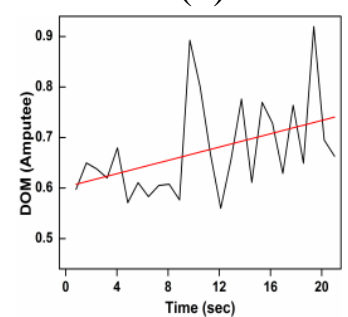

(d)
Figure 3: Representative isometric contraction of Rectus Femoris from (a) Sound leg (b) Amputated leg along with variations of DOM in (c) Sound (d) amputated leg

\section{3b. Multifractal Analysis}

The sEMG signals are segmented into epochs of one second and multifractal features are extracted from each epoch. The degree of multifractality (DOM) for both the sound and amputated leg are 
represented in Figure 4. The slope of DOM in the sound leg and amputated leg is 0.002 and 0.004, respectively. The higher slope in DOM is an indication that variation of multifractality is increasing in the amputated leg. The mean and standard deviation of multifractal features and conventional features are shown in Table 2. The statisical analysis was performed using 2-way ANOVA to analyse the multifractal features in nonfatigue and fatigue condition for both sound and amputated leg. The results showed that the multifractal features are clearly distinguishable in nonfatigue and fatigue condition for both sound and amputated leg ( $p$-value < 0.001) Table 3.

Table 2: Mean (STD) slope and p-value for all features

\begin{tabular}{|l|c|c|c|c|c|c|}
\hline Leg & $\mathbf{H}_{\text {MAX }}$ & $\mathbf{H}_{\text {MIN }}$ & DOM & SOM & $\begin{array}{c}\text { RMS } \\
(\mathbf{m V}) *\end{array}$ & $\begin{array}{c}\text { MDF } \\
(\mathbf{H z})\end{array}$ \\
\hline Sound & -0.005 & -0.003 & 0.002 & 0.003 & 0.002 & -0.105 \\
& $(0.002)$ & $(0.004)$ & $(0.006)$ & $(0.006)$ & $(0.004)$ & $(0.198)$ \\
\hline Amputate & 0.003 & -0.001 & 0.004 & 0.005 & -0.0002 & -0.081 \\
d & $(0.006)$ & $(0.004)$ & $(0.004)$ & $(0.005)$ & $(0.000)$ & $(0.354)$ \\
\hline
\end{tabular}

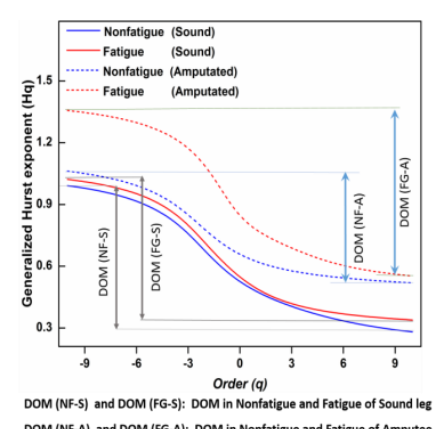

(a)

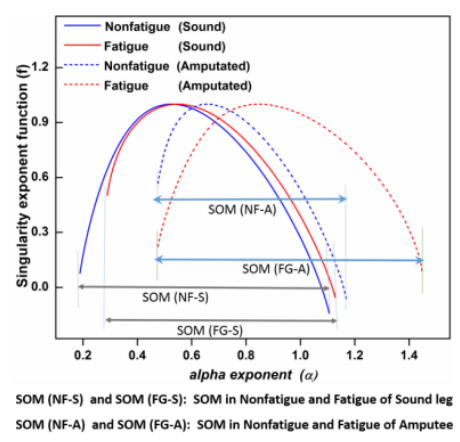

(b)
Figure 4: (a) Generalized Hurst exponentand (b)Multifractal spectrum in nonfatigue (blue lines) and fatigue conditions (red lines) in sound leg (continuous lines) and amputed leg (dotted lines)

Table 3: 2-way ANOVA analysis of features with muscle condition and leg type

\begin{tabular}{|l|c|c|c|c|}
\hline \multirow{2}{*}{ Condition } & \multicolumn{4}{|c|}{ ANOVA ( $p$-value) } \\
\cline { 2 - 5 } & SOM & DOM & RMS & MDF \\
\hline $\begin{array}{l}\text { Muscle condition } \\
\text { (Nonfatigue - Fatigue) }\end{array}$ & VS & VS & NS & NS \\
\hline $\begin{array}{l}\text { Leg type } \\
\text { (Sound - Amputated) }\end{array}$ & NS & NS & NS & NS \\
\hline Interaction & NS & NS & NS & NS \\
\hline
\end{tabular}

VS -Highly statistically significant (p-value < 0.005);

NS - Not statistically significant $(p>0.1)$

\section{Discussion}

Most of the fatigue studies have been done in normal subjects and not much of data is available in patients with clinical conditions. While much is known about the physiological basis of muscle fatigue which is accumulation of metabolites very little is known about EMG consequences of brief fatiguing isometric contraction ${ }^{(9)}$. Under fatigue conditions factors such as recruitment of fast twitch fibres, synchronization of motor units within the muscle, increase in motor unit recruitment and decrease in motor unit firing all lead to spectral changes such as increase in EMG amplitude and decrease in median frequency ${ }^{(10,11,12)}$.

From this study it was observed that the muscle activity in the amputated leg was lower than the sound leg which may be due to the reduction of muscle mass. The amputated leg clearly showed a broad multifractality and shifted to higher values. This may be due to the participation of fewer motor units, lower sEMG activity and increased synchronization of the motor units.

The average slope of strength of multifractality (SOM) in sound and amputated leg are 0.0019 and 0.0047 , respectively. Based on the DOM and SOM values, it is observed that the influence of small fluctuations in sEMG signals are dominant in amputated leg than sound leg. The RMS data is also clearly shows that the muscle activity is reduced in amputated leg.

\section{Conclusions}

The results from this study show that sEMG signals exhibit multifractal behavior in both the sound and amputated leg and the fatigue and nonfatigue segments which were clearly distinguishable using DOM and SOM features. Hence we conclude that multifractal analysis of sEMG signals proved to be better than conventional features (RMS and MDF).

The multifractal analysis of sEMG signals in lower limb amputation subjects can thus help in understanding the muscle dynamics during isometric contraction and fatiguing conditions and thereby prove to be useful to evaluate the effectiveness of rehabilitative or training interventions 


\section{Limitations}

This study is limited to few subjects and a single muscle.

\section{Acknowledgement}

I would like to thank Dr Kiran Marri and the research scholars of NIID lab, Indian Institute of technology, Madras, Chennai without their support and cooperation, this work would not have been completed.

This work was partially presented at RMB Symposium 2016 Colarado, USA.

\section{Source of funding: nil}

Conflict of interest: none declared

\section{References}

1. D. G. Smith, "Transtibial Amputations: Successes and Challenges," inMotion, vol. 13, no. 4, pp. 57-63, 2003.

2. B.D. Chaurasia's Human Anatomy. vol 2, 7th edition 2017, Publisher CBS

3. R. Merletti and P. A. Parker, Electromyography: Physiology, Engineering, and Non-Invasive Applications. Wiley, 2004.

4. M. T. Kristensen, G. Holm, K. KirketerpMøller, M. Krasheninnikoff, and P. Gebuhr, "Very low survival rates after non-traumatic lower limb amputation in a consecutive series: what to do?," Interact. Cardiovasc. Thorac. Surg., vol. 14, no. 5, pp. 543-7, May 2012.

5. G. Wang, X. Ren, L. Li, and Z. Wang, "Multifractal analysis of surface EMG signals for assessing muscle fatigue during static contractions," J. Zhejiang Univ. Sci. A, vol. 8, no. 6, pp. 910-915, May 2007.

6. K. Marri and R. Swaminathan, "Analysis of Biceps Brachii Muscles in Dynamic Contraction Using sEMG Signals and Multifractal DMA Algorithm,” Int. J. Signal Process. Syst., vol. 4, no. 1, pp. 79-85, 2016.

7. H. J. Hermens, B. Freriks, C. DisselhorstKlug, and G. Rau, "Development of recommendations for SEMG sensors and sensor placement procedures.," J. Electromyogr. Kinesiol., vol. 10, no. 5, pp. 361-74, Oct. 2000.

8. G.-F. $\mathrm{Gu}$ and W.-X. Zhou, "Detrending moving average algorithm for multifractals," Phys. Rev. E Stat Nonlin Soft Matter Phys., vol. 82, no. 1, p. 11136, Jul. 2010.

9. J. W. Kantelhardt, S. A. Zschiegner, E. Koscielny-bunde, S. Havlin, A. Bunde, and H. E. Stanley, "Multifractal detrended fluctuation analysis of nonstationary time series," Phys. A Stat. Mech. its Appl., vol. 316, pp. 87-114, 2002

10. Tschoepe BA, Sherwood DE, Wallace SA. Localized muscular fatigue duration, EMG parameters and accuracy of rapid limb movements. J Electromyogr Kinesiol. 1994; 4(4):218-29.

11. Bigland-Ritchie B, Johansson RS, Lippold OCJ. Contractile speed and EMG changes during fatigue of sustained maximal voluntary contractions. J Neurophysiol 1983;50:313-24.

12. Grimby L, TollbackA, Muller U, Larsson L. Fatigue of chronically overused motor units in prior polio patients. Muscle Nerve 1996;19:728-37.

13. Leisman G, Zehausern R, Ferentz A, Tefera T, Zemcov A. EMG effects of fatigue and task repetition on the validity of estimates of strong and weak muscles in applied kinesiological muscle-testing procedures. Percept Mot Skills 1995;80:963-77. 